Enferm Bras 2020;19(4);290-301

https://doi.org/10.33233/eb.v19i4.3100

\title{
ARTIGO ORIGINAL \\ Conhecimento e práticas de pessoas com diabetes mellitus sobre a terapêutica medicamentosa e suas complicações agudas
}

Lilian Cristiane Gomes, D.Sc. ${ }^{\star}$, Luciana Castelar Tsuda, D.Sc. ${ }^{* *}$, Ana Emilia Pace, D.Sc. ${ }^{\star * *}$

Enfermeira, Coordenadora e docente do Curso de Enfermagem do Centro Universitário da Fundação Educacional Guaxupé (UNIFEG), Guaxupé/MG, ${ }^{* \star}$ Enfermeira Especialista em Laboratório na Escola Superior de Agricultura Luiz de Queiroz da Universidade de São Paulo (ESALQ / USP), Piracicaba/SP, ${ }^{* * * E n f e r m e i r a, ~ P r o f e s s o r ~ A s s o c i a d o ~ S e ̂ n i o r ~ d o ~ D e p a r t a m e n t o ~ d e ~}$ Enfermagem Geral e Especializada da EERP-USP, Ribeirão Preto/SP

Recebido em 9 de setembro de 2019; aceito em 20 de agosto de 2020.

Correspondência: Lilian Cristiane Gomes, Avenida Dona Floriana, 463 Centro 37800-000 Guaxupé MG

Lilian Cristiane Gomes: liliancristianegomes@yahoo.com.br

Luciana Castelar Tsuda: lucianacastelar@yahoo.com.br

Ana Emilia Pace: aepace@eerp.usp.br

\section{Resumo}

Objetivo: Identificar o conhecimento e as práticas acerca da terapêutica medicamentosa e suas complicações agudas entre as pessoas com diabetes mellitus. Métodos: Estudo seccional, de abordagem quantitativa, com uma amostra de 46 adultos em seguimento ambulatorial em uma unidade de atenção terciária à saúde, no interior paulista. A coleta de dados foi realizada por meio de entrevista face a face, com o uso de instrumento semiestruturado, elaborado a partir de estudos prévios, e simulação do processo de preparo e administração da insulina. Resultados: Houve maior frequência de sexo feminino, casados/amasiados, inativos no mercado de trabalho, procedentes de Ribeirão Preto e/ou região, com escolaridade média de 5,6 anos de estudo, tempo médio de diagnóstico de 16,4 anos e em insulinoterapia. Quanto ao conhecimento e às práticas relativas à terapêutica medicamentosa, observaram-se fragilidades no processo de preparo e administração da insulina e na prevenção de suas complicações agudas, como a hipoglicemia. Conclusão: Os resultados reforçam a necessidade de monitoramento contínuo durante o curso da doença, no que se refere, em particular, à insulinoterapia, de modo a proporcionar um tratamento seguro e eficaz.

Palavras-chave: diabetes mellitus, conhecimento do paciente sobre a medicação, cuidados de enfermagem.

\footnotetext{
Abstract

Knowledge and practices of people with diabetes mellitus about the drug therapy and their acute complications

Objective: To identifying knowledge and practices about drug therapy and its acute complications among people with diabetes mellitus. Methods: This is a sectional study, with a quantitative approach, and the sample consisted of 46 adults in outpatient follow-up at a tertiary health care unit in the interior of São Paulo, Brazil. Data collection was performed through face-to-face interviews, using a semi-structured instrument, elaborated from previous studies, and simulation of the insulin preparation and administration process. Results: There was a higher frequency of female patients, married or living with a partner, retired or pensioners, residing in a municipality close to where the study was implemented, with low educational and socioeconomic level. The sample had a long disease duration, with insulin therapy. Regarding the knowledge and practices about the drug treatment, weaknesses were observed in the process of preparation and administration of insulin and in the prevention of its acute complications, such as hypoglycemia. Conclusion: The results reinforce the need for continuous monitoring during the course of the disease, particularly with regard to insulin therapy, in order to provide a safe and effective treatment.
} 
Keywords: diabetes mellitus, patient medication knowledge, nursing care.

\section{Resumen \\ Conocimientos y prácticas de personas con diabetes mellitus sobre la farmacoterapia y sus complicaciones agudas}

Objetivo: Identificar conocimientos y prácticas sobre la farmacoterapia y sus complicaciones agudas en personas con diabetes mellitus. Métodos: Estudio transversal, con abordaje cuantitativo, con una muestra de 46 adultos en seguimiento ambulatorio en una unidad terciaria de salud del interior de São Paulo. La recolección de datos se realizó mediante entrevistas presenciales, utilizando un instrumento semiestructurado, elaborado a partir de estudios previos, y simulación del proceso de preparación y administración de la insulina. Resultados: Hubo una mayor frecuencia de mujeres, casadas / convividas, inactivas en el mercado laboral, de Ribeirão Preto y / o región, con una escolaridad promedio de 5,6 años de estudio, tiempo medio de diagnóstico de 16,4 años y en terapia con insulina. En cuanto a conocimientos y prácticas relacionadas con la farmacoterapia, se observaron debilidades en el proceso de preparación y administración de la insulina y en la prevención de sus complicaciones agudas, como la hipoglucemia. Conclusión: Los resultados refuerzan la necesidad de un seguimiento continuo durante el curso de la enfermedad, en lo que respecta, en particular, a la terapia con insulina, con el fin de proporcionar un tratamiento seguro y eficaz.

Palabras-clave: diabetes mellitus, conocimiento del paciente sobre la medicación, cuidado de enfermería.

Introdução

O Diabetes Mellitus (DM) é uma doença crônica que demanda contínuo cuidado clínico e educação para o seu automanejo, a fim de prevenir as complicações agudas e reduzir o risco de complicações a longo prazo [1]. No curso da doença, a meta principal é que a pessoa assuma o seu próprio cuidado [2] sendo fundamental, para tanto, a aquisição de conhecimentos sobre a doença, tratamento e seu controle, bem como o desenvolvimento de habilidades para uma prática medicamentosa segura, de acordo com as necessidades e características individuais [3].

Atualmente, uma ampla variedade de agentes antidiabéticos orais (AAOs), com distintos mecanismos de ação, está disponível para o tratamento do DM tipo 2 (DM2) [4]. Embora o uso dos AAOs (monoterapia ou associações) seja o tratamento medicamentoso mais frequentemente prescrito [4,5], estudos realizados entre adultos brasileiros com DM apontam a falta de conhecimento das pessoas em relação aos AAOs, especialmente no que se refere ao nome do fármaco, horário de tomada e mecanismo de ação $[6,7]$.

Em relação ao uso de insulina exógena no tratamento do DM2, apesar de ser a medicação hipoglicemiante mais efetiva disponível até o momento, não existindo limitações de dosagens quanto ao seu efeito terapêutico e nem contraindicações para o seu uso, observa-se que a insulinização é menos frequente do que deveria, e seu início tende a ser tardio [4]. Além disso, estudos desenvolvidos entre pessoas com DM, acompanhadas em unidades básicas de atenção primária à saúde, apontam erros e dificuldades das pessoas em relação aos procedimentos inerentes à administração de insulina $[8,9]$.

A literatura aborda algumas barreiras relacionadas à terapêutica medicamentosa, tais como a idade avançada, a baixa escolaridade, as limitações físicas e/ou funcionais [10,11] e os aspectos psicológicos, sobretudo as crenças errôneas e os mitos por parte das pessoas e suas famílias [12,13], os quais precisam ser reconhecidos para se promover o autocuidado.

A hipoglicemia é um episódio frequente no início do tratamento com a insulina ou AAO, especialmente a classe das sulfonilureias, ou pode ocorrer em decorrência de práticas inadequadas relacionadas a essas modalidades de tratamento $[4,14]$. Portanto, recomenda-se que a pessoa com DM esteja ciente da possibilidade dessa ocorrência, saiba reconhecer sinais e sintomas e receba as orientações necessárias para a reversão do quadro [4].

Ressalta-se que o tratamento medicamentoso do DM, embora complexo, objetiva a obtenção e a manutenção de um bom controle glicêmico, dada a sua importância para o manejo da doença e prevenção de suas complicações crônicas. Dessa forma, o tratamento medicamentoso deverá ser conduzido de forma segura e para a máxima efetividade terapêutica [12].

Mediante o exposto, avaliar como as pessoas com DM conduzem seu tratamento na vida diária torna-se relevante e constitui a base para a realização de intervenções educativas que possam habilitar a pessoa e sua família para a o uso seguro e eficaz dos medicamentos. No 
Brasil, são escassos os estudos que avaliaram tal situação "de modo realístico", por meio de demonstração. Portanto, o presente estudo tem por objetivo descrever o conhecimento e as práticas relativas à terapêutica medicamentosa, bem como as informações sobre sinais, sintomas, prevenção e tratamento da hipoglicemia, uma vez que esta última pode relacionar-se com essa terapêutica.

\section{Material e métodos}

Trata-se de um estudo seccional, descritivo e de abordagem quantitativa, realizado em unidade ambulatorial de um hospital-escola do interior paulista, entre setembro de 2010 e março de 2011.

A amostra do estudo foi por conveniência e selecionada por meio da revisão semanal dos prontuários das pessoas agendadas para atendimento com a equipe de saúde, mediante os critérios de inclusão/exclusão. Como critérios de inclusão foram considerados: adultos com DM, de ambos os sexos, em tratamento medicamentoso com insulina, AAOs e/ou associações, e que apresentassem capacidade de manter diálogo. Foram excluídos aqueles que apresentavam complicações crônicas em estágio avançado, tais como tratamento dialítico, amaurose, sequelas de acidente vascular encefálico e/ou de insuficiência cardíaca, amputação prévia e/ou úlcera ativa em extremidade inferior, e em uso de cadeira de rodas ou maca.

O convite para a participação no estudo foi realizado verbalmente, na sala de espera, enquanto as pessoas aguardavam o atendimento. Após a apresentação dos objetivos do estudo e o esclarecimento sobre 0 anonimato da participação, todas as pessoas abordadas concordaram em participar e foram conduzidas a uma sala privativa, na qual foi entregue o Termo de Consentimento Livre e Esclarecido (TCLE), que foi lido em voz alta por uma das pesquisadoras, e solicitada a assinatura do mesmo ao final da leitura. Dessa forma, a amostra ficou constituída por 46 pessoas.

A coleta de dados foi realizada por meio de entrevista face a face. Utilizou-se um instrumento semiestruturado, baseado em estudos anteriores $[9,10,15]$, para obtenção das variáveis sociodemográficas, clínicas e de tratamento medicamentoso. O tempo médio de duração das entrevistas foi de 55 minutos.

Durante a entrevista, foram oferecidos frascos de insulina e seringas com agulhas e solicitado, àqueles que realizam a autoaplicação, que demonstrassem a aspiração da dose. Os respectivos prontuários foram verificados para a comparação entre a dose prescrita e a administrada.

Em relação aos locais referidos para a aplicação da insulina, foram considerados totalmente adequados aqueles descritos na literatura (regiões laterais direita e esquerda do abdome, tomando-se a distância de quatro centímetros ao redor da cicatriz umbilical; face posterior dos braços, na porção compreendida entre a axila e o cotovelo; região anterolateral externa das coxas e quadrante superior lateral externo das nádegas) [4,16]; inadequados quando utilizadas outras regiões dos locais citados anteriormente (região lateral dos braços, por exemplo) ou outras partes do corpo (antebraços, panturrilhas, entre outros). Referente ao rodízio dos locais de aplicação, foi considerado adequado quando realizado dentro da mesma região corporal até completar os locais disponíveis, tendo o cuidado de manter a distância mínima de um centímetro entre uma aplicação e outra; e inadequado quando cada aplicação é feita em distintos locais, sem atentar-se ao rodízio, ou seja, de forma indiscriminada $[4,16]$.

Quanto ao armazenamento da insulina no domić́lio, em geladeira, foi considerado adequado quando utilizadas a última prateleira e/ou a gaveta inferior; e inadequado quando utilizada qualquer outra parte do equipamento, especialmente a porta, o congelador ou a prateleira logo abaixo deste [4].

Para o descarte de seringas e agulhas, considerou-se adequado quando realizado em recipientes de plástico rígido, com boca larga e tampa rosqueável $[4,16]$, sendo os mesmos encaminhados aos serviços de saúde, e inadequado, quando os insumos são desprezados no lixo doméstico. Destaca-se que os erros identificados durante as entrevistas, tanto em relação ao uso de AAOs, quanto a qualquer etapa do processo de administração de insulina, foram corrigidos de forma imediata e comunicados aos familiares posteriormente.

Os dados coletados foram armazenados no programa MS-Excel, com dupla digitação e validação dos mesmos. Posteriormente, o banco de dados foi exportado para o programa Statistical Package for Social Science (SPSS), versão 17.0, para análise exploratória univariada, empregando-se a estatística descritiva, com medidas de tendência central (média e mediana) e de variabilidade (desvio-padrão), bem como medidas de frequência das variáveis. 
O presente estudo integra o projeto intitulado "Habilidade para o autocuidado das pessoas com diabetes mellitus", aprovado pelo Comitê de Ética em Pesquisa do Hospital das Clínicas da Faculdade de Medicina da Universidade de São Paulo, sob o processo nํ4216/2010.

Resultados

Na caracterização sociodemográfica da amostra, a média de idade e o respectivo desviopadrão (DP) foram de 59,4 anos (DP=13,2). Dos 46 participantes, $24(52,4 \%)$ eram do sexo feminino, a maioria $(60,9 \%)$ eram casados/amasiados, inativos no mercado de trabalho $(82,6 \%)$, procedentes de Ribeirão Preto e/ou região (73,9\%), com escolaridade média de 5,6 anos de estudo $(D P=4,2)$ e média de renda familiar mensal de $R \$ 1.333,41 \quad(D P=556,52)$.

O tempo de diagnóstico foi, em média, de 16,4 anos ( $(\mathrm{PP}=8,0)$ e $43(93,5 \%)$ compareciam aos retornos agendados pelo serviço de saúde. Entre os 46 participantes, $25(54,3 \%)$ referiram uso de AAOs. Destes, 23 (92\%) o fazem em monoterapia. O tempo médio de uso foi de 9,8 anos $(\mathrm{DP}=6,4)$, e a frequência diária de tomada foi, em média, de 2,5 vezes ao dia. A classe medicamentosa de maior frequência $(80 \%)$, independentemente de associações, foi a das biguanidas. Quando questionado sobre o horário de tomada dos AAOs, dois (50\%), entre os quatro entrevistados que fazem uso de sulfonilureia, afirmaram tomá-la após as refeições; entre os 22 que utilizam biguanida, quatro $(18,2 \%)$ relataram tomá-la antes das refeições (Tabela I).

Tabela I - Distribuição numérica e percentual da amostra estudada referente ao tratamento medicamentoso com AAOs, Ribeirão Preto/SP, 2012.

\begin{tabular}{lll}
\hline Variáveis (N = 46) & $\mathbf{N}$ & $\%$ \\
\hline Uso de AAOS & & \\
\hline Sim & 25 & 54,3 \\
Não & 21 & 45,7 \\
\hline Associação de AAOs (N=25) & 2 & 8 \\
\hline Sim & 23 & 92 \\
Não & & \\
\hline Tipo de AAOs (N=25) & 02 & 8 \\
Somente sulfonlurela & 20 & 80 \\
Somente biguanida & 02 & 8 \\
Sulfonilureia + biguanida & 01 & 4 \\
Toglitazone & 01 & 25 \\
\hline Horário de tomada da sulfonilu reia (N=4) & & \\
\hline Antes das refeições & 01 & 25 \\
Durante as refeições & 02 & 50 \\
Após as refeiços & & \\
\hline Horário de tomada da biguanida (N=22) & 04 & 18,2 \\
\hline Antes das refeições & 02 & 9,1 \\
Durante as refeições & 16 & 72,7 \\
Após as refeiços & & \\
\hline Horário de tomada da toglitazone (N=1) & 01 & 100 \\
\hline Após as refeições & & \\
\hline
\end{tabular}

Referente à insulinização, entre os 46 participantes, $42(91,3 \%)$ referiram uso de insulina associada ou não ao uso de AAOs. O tempo médio de uso foi de 8,9 anos (DP = 7,7), e a frequência diária de aplicação foi, em média, de duas vezes ao dia (DP $=0,9)$. Para a administração de insulina, 41 (97,6\%) relataram uso de seringas e agulhas, e $1(2,4 \%)$ relatou 0 uso de caneta injetora.

Quanto aos tipos de insulina prescritos, $18(42,9 \%)$ referiram uso de insulina humana $\mathrm{NPH}$ associada à insulina Regular (R), e esse mesmo percentual foi encontrado para o uso isolado de insulina NPH. O uso de NPH e R, bem como NPH e Lispro, em aplicações distintas, foi referido, respectivamente, por três $(7 \%)$ e um $(2,4 \%)$ dos 42 participantes. O uso de prémistura foi declarado por um $(2,4 \%)$ participante. Destaca-se que uma pessoa $(2,4 \%)$ desconhecia o tipo de insulina em uso.

Aproximadamente $36 \%$ dos participantes referiram depender totalmente do auxílio de outra pessoa para o processo de administração da insulina, e o principal motivo para essa dependência foi medo e/ou insegurança para executar as etapas da técnica de aplicação. 
Entretanto, cerca de $91 \%$ dos responsáveis pela administração insulínica não estavam presentes na consulta.

Entre os 27 (64,3\%) entrevistados que realizavam a autoaplicação, 16 (59,3\%) aspiraram corretamente a dose de insulina, conforme a prescrição médica. Dos $41(97,6 \%)$ participantes que utilizavam seringas e agulhas para a administração insulínica, respectivamente, 31 (75,6\%) e $30(73,2 \%)$ as reutilizavam. A frequência de reutilização de seringas e agulhas variou de uma a seis vezes e aproximadamente $60 \%$ dos participantes as reutilizaram entre duas e quatro vezes. Outros aspectos relacionados à insulinoterapia estão apresentados nas Tabelas II e III.

Tabela II - Distribuição numérica e percentual da amostra estudada, segundo a participação da própria pessoa e/ou de outra no processo de administração de insulina, Ribeirão Preto/SP, 2012.

\begin{tabular}{|c|c|c|}
\hline Variáveis $(\mathrm{N}=42)$ & $\mathbf{N}$ & $\%$ \\
\hline \multicolumn{3}{|l|}{ Pessoa que aspira e/ou aplica in sulina } \\
\hline a própria pessoa & 20 & 47,6 \\
\hline outra pessoa & 15 & 35,7 \\
\hline ambas & 7 & 16,7 \\
\hline \multicolumn{3}{|l|}{$\begin{array}{l}\text { Motivo da participação de outra pessoa no processo de } \\
\text { administração de insulina }\end{array}$} \\
\hline medo/insegurança & 12 & 54,5 \\
\hline preferência pessoal/comodidade & 4 & 18,2 \\
\hline dificuldade visual para aspirar a dose & 3 & 13,7 \\
\hline dificuldade manual para aplicar & 2 & 9,1 \\
\hline por orientação profissional & 1 & 4,5 \\
\hline \multicolumn{3}{|l|}{$\begin{array}{l}\text { Presen ça, na con sulta, da pessoa respon sável pela } \\
\text { administração de insulina }(\mathrm{N}=22)\end{array}$} \\
\hline $\begin{array}{l}\text { Sim } \\
\text { Não }\end{array}$ & 20 & $\begin{array}{r}9,1 \\
90,9\end{array}$ \\
\hline \multicolumn{3}{|l|}{$\begin{array}{l}\text { Motivo da ausência da pessoa respon sável pela } \\
\text { administração de insulina, na consulta }(\mathrm{N}=20)\end{array}$} \\
\hline compromisso de trabalho & 9 & 45 \\
\hline por ser profissional de saúde da UBS/farmácia & 2 & 10 \\
\hline problemas familiares & 2 & 10 \\
\hline dificuldade de transporte & 1 & 5 \\
\hline não soube informar & 6 & 30 \\
\hline
\end{tabular}

Visto que a hipoglicemia é uma das complicações agudas mais comuns da insulinoterapia e do uso de sulfonilureias, os participantes do presente estudo foram questionados a respeito do reconhecimento de seus sinais e sintomas, como preveni-la e tratála. Observou-se que $41(89,1 \%)$ participantes sabem reconhecer os sinais e sintomas de hipoglicemia, dois $(4,3 \%)$ não sabem e três $(6,5 \%)$ relataram nunca ter apresentado os sintomas; $29(63 \%)$ e $41(89,1 \%)$ sabem, respectivamente, prevenir e tratar a hipoglicemia.

Tabela III - Distribuição numérica e percentual da amostra estudada referente ao processo de administração de insulina, Ribeirão Preto/SP, 2012. 


\begin{tabular}{|c|c|c|}
\hline Variáveis ( $\mathrm{N}=42)$ & $\mathrm{N}(\%)$ & Média (DP) \\
\hline \multicolumn{3}{|c|}{ Aspira a do se correta, conforme a prescricão ( $\mathrm{N}=27$ ) } \\
\hline $\operatorname{Sim}_{\text {Não }}$ & $\begin{array}{l}16(59,3) \\
11(40,7)\end{array}$ & \\
\hline \multicolumn{3}{|l|}{ Injeta ar no frasco para facilitar a aspiração } \\
\hline $\operatorname{Sim}_{\text {Não }}$ & $\begin{array}{l}16(59,3) \\
11(407)\end{array}$ & \\
\hline \multicolumn{3}{|l|}{ Locais referid os para a aplicação de insulina } \\
\hline $\begin{array}{l}\text { Adequados } \\
\text { Inadequados }\end{array}$ & $\begin{array}{l}6(14,3) \\
36(85,8)\end{array}$ & \\
\hline \multicolumn{3}{|l|}{ Realização de prega subcutânea } \\
\hline $\operatorname{Sim}_{\text {Não }}$ & $\begin{array}{l}35(83,3) \\
7(16,7)\end{array}$ & \\
\hline \multicolumn{3}{|c|}{$\begin{array}{l}\text { Seringa com graduação ad equad a à do se prescrita e comprimento } \\
\text { adequado da agulha }\end{array}$} \\
\hline $\begin{array}{l}\text { Sim } \\
\text { Não }\end{array}$ & $\begin{array}{l}41(97,6) \\
1(2,4)\end{array}$ & \\
\hline \multicolumn{3}{|l|}{ Rodizio dos locais para a aplicação de insulina } \\
\hline $\begin{array}{l}\text { Adequado } \\
\text { Inadequado } \\
\text { Não realiza rodizio }\end{array}$ & $\begin{array}{l}15(35,7) \\
22(52,4) \\
5(119)\end{array}$ & \\
\hline \multicolumn{3}{|l|}{ Armazenamento da insulina } \\
\hline $\begin{array}{l}\text { Adequado } \\
\text { Inadequado }\end{array}$ & $\begin{array}{l}15(35,7) \\
27(64,3)\end{array}$ & \\
\hline \multicolumn{3}{|l|}{ Reutilizaçáo de seringas $(\mathrm{N}=41)$} \\
\hline $\begin{array}{l}\text { Sim } \\
\text { Não }\end{array}$ & $\begin{array}{l}31(75,6) \\
10(24,4)\end{array}$ & \\
\hline $\begin{array}{l}\text { Número de vezes que reutilizou cada seringa } \\
\text { Reutilização de agulhas }(\mathrm{N}=41)\end{array}$ & & $3,1(1,2)$ \\
\hline $\begin{array}{l}\text { Sim } \\
\text { Não }\end{array}$ & $\begin{array}{l}30(73,2) \\
11(26,8)\end{array}$ & \\
\hline Número de vezes que reutilizou cada agulha & & $3,0(1,1)$ \\
\hline \multicolumn{3}{|l|}{ Descarte de seringas e/ou agulhas } \\
\hline $\begin{array}{l}\text { Adequado } \\
\text { Inadequado }\end{array}$ & $\begin{array}{l}24(57,1) \\
18(42,9)\end{array}$ & \\
\hline
\end{tabular}

\section{Discussão}

A amostra estudada constituiu-se por adultos, em sua maioria, do sexo feminino, de baixa escolaridade e renda, vivendo com companheiro(a), procedentes de Ribeirão Preto e/ou região, com longo tempo de doença e em insulinoterapia. Esses resultados, em geral, assemelham-se aos de outras pesquisas realizadas no local de estudo $[17,18]$, podendo caracterizar o perfil das pessoas que procuram por atendimento médico em Ribeirão Preto.

Em relação ao tratamento medicamentoso, $42(91,3 \%)$ referiram uso de insulina associado ou não ao uso de AAOs; o tempo médio de uso da insulina e dos AAOs foi cerca de nove a dez anos, respectivamente. Estudo retrospectivo realizado numa amostra de 5.403 adultos suecos com DM2, cujo objetivo foi analisar o período de tempo entre o uso de AAOs e o início da insulinização, bem como os fatores associados com a prescrição de insulina, revelou que após seis anos de uso de AAOs, cerca de $25 \%$ da amostra iniciou a insulinoterapia e, após 10 anos, essa taxa aumentou para $42 \%$. A probabilidade de insulinização foi maior entre as pessoas com menos de 65 anos, uso de mais de um antidiabético oral e com pior controle glicêmico [19].

Quanto ao horário de tomada dos AAOs, entre os quatro entrevistados que fazem uso de sulfonilureia, dois $(50 \%)$ afirmaram tomá-la após as refeições, e entre os 22 que utilizam biguanida, quatro $(18,2 \%)$ relataram tomá-la antes das refeições. Esses achados sugerem falta de conhecimento das pessoas com DM em relação ao horário de tomada dos medicamentos prescritos, corroborando outros estudos descritivos [5-6]. Ao considerar que no DM as pessoas são as principais responsáveis pelo seu próprio cuidado [2,3], a falta de entendimento acerca do horário de tomada dos medicamentos pode se constituir em uma barreira para a adesão ao tratamento e comprometer o alcance dos resultados clínicos desejados. Cabe aos profissionais de saúde identificar as dificuldades relacionadas ao uso dos medicamentos e estabelecer estratégias para minimizá-las, uma vez que a adesão ao tratamento deve ser vista como uma 
atividade conjunta na qual a pessoa não somente obedece às orientações médicas, mas entende, concorda com e adota o regime prescrito [20].

Estudo sobre as interações entre AAOs e alimentos aponta que, referente às sulfonilureias, o horário recomendado para a sua administração é 30 minutos antes das refeições [5], visto que o seu mecanismo de ação consiste em aumentar a secreção de insulina [4-5] e, dessa forma, a pessoa obterá uma quantidade satisfatória desse hormônio para reduzir a glicemia proveniente da alimentação [5]. Quanto às biguanidas, recomenda-se administrá-las de 15 a 20 minutos após a alimentação [5], uma vez que ela reduz a produção de glicose hepática e apresenta, ainda que discretamente, uma ação sensibilizadora periférica dos receptores de insulina [4-5].

Referente à insulinização, verificou-se a dependência do auxílio de outra pessoa para os procedimentos de preparo e/ou administração da insulina em 15 (35,7\%) participantes, semelhante a outros estudos nacionais descritivos [8,10]. Entretanto, sempre que possível, a insulina deve ser administrada pela própria pessoa, uma vez que a autoaplicação favorece a autonomia e a adesão ao tratamento [1].

Quando questionados sobre os motivos para a participação de outra pessoa no processo de administração da insulina, $12(54,5 \%)$ referiram medo e/ou insegurança para a autoaplicação. A literatura aponta que essa é uma das principais barreiras para o tratamento insulínico, além de crenças errôneas e mitos por parte das pessoas e de seus familiares [12,13]. Nesse contexto, ressaltam-se as habilidades necessárias aos profissionais de saúde para identificar a falta de conhecimento, identificar e clarificar crenças, atitudes, comportamentos e sentimentos sobre a insulinoterapia, com o objetivo de auxiliar as pessoas com DM a superar o medo e/ou a insegurança. Essas habilidades incluem a escuta ativa, a empatia e o incentivo à autonomia das pessoas $[1,4]$.

Entre os que realizavam a autoaplicação, 59,3\% aspiraram a dose correta de insulina, conforme prescrito em prontuário. Administrar uma dose menor ou maior que a prescrita pode ocasionar, respectivamente, um controle glicêmico inadequado ou episódios de hipoglicemia [21]. Baixa acuidade visual, falta de destreza manual e idade avançada podem favorecer erros de dosagem $[10,11,22]$. Estudo realizado entre adultos brasileiros com DM, acompanhados pela Estratégia Saúde da Família, cujo objetivo foi comparar a dose aspirada de insulina com a dose prescrita, encontrou que $36,1 \%$ dos participantes cometeram divergências na aspiração da dose, e as variáveis preditoras foram o sexo (feminino), a idade ( $>60$ anos) e a escolaridade $(<8$ anos de estudo) [11]. Estudo brasileiro multicêntrico, que objetivou identificar e quantificar a ocorrência de erros técnicos durante a autoadministração de insulina, mostrou resultados semelhantes quanto à idade, além de maior ocorrência de erros entre os pacientes com DM2, menor tempo de insulinização e que não praticavam rodízio dos locais de aplicação e/ou o praticavam de forma incorreta [21].

A obtenção do controle glicêmico depende da capacidade de a pessoa aspirar e administrar quantidades exatas de insulina [22]. Para aspirar a dose correta, recomenda-se a injeção de ar no frasco de insulina na mesma quantidade da dose prescrita, uma vez que esse procedimento evita a formação de vácuo no frasco e facilita a aspiração $[4,9,16]$. Entre os pacientes que aspiraram corretamente a dose de insulina (59,3\%), todos injetaram ar no frasco previamente à aspiração. Esse achado diverge dos de outros dois estudos brasileiros: o primeiro se propôs a descrever os erros e os acertos mais comuns da técnica de autoaplicação de insulina com seringas descartáveis [9], e o segundo, investigou a competência de idosos com DM para a autoaplicação de insulina [22], sendo que em ambos os estudos a maioria dos participantes não realizava tal procedimento $[9,22]$.

Quanto aos locais para a aplicação de insulina, somente seis $(14,3 \%)$ pacientes referiram regiões corporais corretas, conforme preconizado pela literatura. A insulina deve ser aplicada no tecido subcutâneo, uma vez que a extensa rede de capilares sanguíneos possibilita a absorção gradativa do medicamento e garante o perfil farmacocinético descrito pelo fabricante [4]. Ressalta-se que os estudos brasileiros que avaliaram o processo de administração de insulina não abordaram este último tópico, o que limita as comparações deste resultado. Outro aspecto importante é a realização do rodízio dos locais de aplicação de insulina para que o tratamento seja seguro e eficaz, além de prevenir a lipodistrofia e consequente hiperglicemia $[4,16,21]$. No presente estudo, $22(52,4 \%)$ realizavam o rodízio de modo inadequado, ou seja, de forma indiscriminada; e cinco $(11,9 \%)$ não o realizam, semelhante ao estudo brasileiro multicêntrico citado previamente [21]. Para o planejamento do rodízio, devem-se considerar a velocidade de absorção da insulina em cada região corporal, os tipos de insulina em uso, o número de aplicações e respectivos horários, além das atividades de vida diária [4]. O rodízio considerado 
correto é aquele realizado de forma sistemática em uma mesma região corporal, ao invés de utilizar distintas regiões a cada aplicação, contribuindo para minimizar a variabilidade de absorção da insulina, ao longo de um período $[4,16]$. Dessa forma, uma mesma região corporal pode apresentar, em média, de sete a 12 pontos de aplicação, com a distância de um centímetro entre eles. Após aplicar em um desses pontos, recomenda-se evitá-los por 14 dias, tempo necessário para a sua cicatrização e prevenção da lipodistrofia [4].

Outro aspecto importante da insulinoterapia é a realização da prega subcutânea. No presente estudo, $35(83,3 \%)$ participantes afirmaram realizá-la, percentual superior ao encontrado pelo estudo brasileiro multicêntrico (30\%), citado previamente [21]. A prega subcutânea aumenta a possibilidade de a insulina ser injetada corretamente no tecido subcutâneo. Deve ser feita utilizando apenas os dedos indicador e polegar, uma vez que, quando realizada com todos os dedos, aumenta-se o risco de injeção intramuscular $[4,16]$. Além disso, a manutenção da prega durante a aplicação de insulina, aguardando, no mínimo, cinco a 10 segundos, respectivamente, para seringa e caneta, antes de se retirar a agulha, reduz o risco de injeção intramuscular e evita o extravasamento de insulina, contribuindo para um melhor controle glicêmico [4,21]. Ressalta-se que a prega subcutânea deve ser realizada em todas as aplicações quando utilizadas agulhas de 12,7 milímetros $(\mathrm{mm})$ e de $8 \mathrm{~mm}$, sendo dispensável para as agulhas de 4, 5 e $6 \mathrm{~mm}[4]$.

A apropriada graduação da seringa e o comprimento adequado da agulha também são fatores decisivos para uma injeção segura e eficaz, com desconforto mínimo [4]. Ao contrário do estudo brasileiro multicêntrico [21], verificou-se que a maioria dos pacientes utilizava seringas com graduação apropriada à dose prescrita. Quanto ao comprimento da agulha, até 2010 , ano no qual se deu início o presente estudo, a recomendação era feita de acordo com o índice de massa corporal (IMC). Dessa forma, o comprimento da agulha em uso foi considerado adequado para $41(97,6 \%)$ participantes. Entretanto, em 2011, novas recomendações foram publicadas a partir do estudo The Third Injection Tecnique Workshop in Athens (TITAN), realizado em 2009 em Atenas, na Grécia [16]. Os seus resultados mostraram que a espessura da pele dos adultos raramente ultrapassa $3 \mathrm{~mm}$ e não sofre influência de características como idade, sexo, raça, tipo físico ou IMC. As pessoas obesas possuem a pele com espessura semelhante à de pessoas magras; em contrapartida, o tecido subcutâneo varia com o sexo, idade, área corporal e IMC $[4,16]$.

As principais agulhas disponíveis no mercado brasileiro $(12,7 \mathrm{~mm}$ e $8 \mathrm{~mm})$, anteriormente recomendadas para a aplicação de insulina, agora são consideradas longas, respectivamente, para muitos adultos e para a maioria das crianças, aumentando o risco de injeção intramuscular. As novas recomendações apontam o uso de agulhas mais curtas $(4,5$ e $6 \mathrm{~cm})$ pelos adultos, inclusive os obesos. Cabe destacar que a decisão quanto ao comprimento da agulha deve ser feita de forma individualizada, em parceria com o paciente, considerando os aspectos físicos, emocionais, farmacológicos e os dispositivos para a aplicação (seringa ou caneta) [4,16].

Referente ao armazenamento da insulina em geladeira, 27 (64,3\%) participantes referiram locais inadequados de conservação, tais como a porta e a parte superior do equipamento, indicando a necessidade de maiores esclarecimentos sobre essa questão. Outros estudos obtiveram achados semelhantes $[9,22]$.

Existem diferenças entre o frasco de insulina em uso e o lacrado quanto à conservação e validade. Recomenda-se que o frasco aberto ou a caneta descartável em uso sejam mantidos na parte inferior da geladeira doméstica (de $2^{\circ}$ a $8^{\circ} \mathrm{C}$ ) ou em temperatura ambiente, desde que não ultrapasse $30^{\circ}$ C. Após quatro semanas da data de abertura e início do uso, deve-se desprezar o frasco e/ou a caneta descartável, mesmo que ainda reste insulina [4,9,22]. Esta última recomendação também é válida para a caneta recarregável com o refil em uso, e a mesma deve ser conservada em temperatura ambiente, desde que não ultrapasse $30^{\circ} \mathrm{C}$ [4].

Os fabricantes advertem que a conservação da caneta recarregável em geladeira pode danificar o seu mecanismo interno e até mesmo interferir no registro da dose correta. Em relação ao frasco e à caneta descartável lacrados, os mesmos devem ser mantidos na parte inferior da geladeira e sua validade pode variar de dois a três anos, a partir da data de fabricação [4]. Vale ressaltar que abaixo de $2^{\circ} \mathrm{C}$, a insulina congela e perde seu efeito. Por essa razão, não deve ser acondicionada na parte superior da geladeira. Da mesma forma, não deve ser conservada na porta do equipamento devido à maior variação de temperatura e à mobilidade excessiva do frasco a cada abertura da porta $[4,9]$.

Quando questionados a respeito da reutilização de seringas e agulhas, aproximadamente $70 \%$ dos participantes afirmaram realizar tal prática, e a maior frequência de reutilização $(60 \%)$ foi a de duas a quatro vezes, resultado este semelhante ao de outros estudos 
nacionais e descritivos $[15,23,24]$. Embora o frasco de insulina contenha aditivos bacteriostáticos (fenol e metacresol) que inibem o crescimento bacteriano dentro do mesmo [15], a reutilização de seringas e agulhas pode favorecer a ocorrência de infecção, uma vez que a esterilidade desses insumos é garantida pelos fabricantes somente no primeiro uso. Além disso, devido ao frequente reaproveitamento, pode ocorrer a perda de nitidez da escala de graduação da seringa, da lubrificação da agulha e da afiação, risco de quebra ou danificação da agulha, alterações no bisel, cristalização do resíduo de insulina no lúmen da agulha e consequente obstrução do fluxo de insulina na próxima aplicação $[4,15]$.

No Brasil, a legislação vigente proíbe o reprocessamento e/ou reesterilização de materiais descartáveis, após o primeiro uso [25]. Entretanto, na prática clínica, observa-se que a reutilização de seringas e agulhas pelas pessoas com DM é bastante comum, especialmente entre aquelas com dificuldades financeiras, visto que a distribuição de insumos pelos serviços públicos de saúde parece ser irregular ou insuficiente às necessidades diárias de aplicações de insulina $[23,24,26]$.

Dessa forma, a reutilização desses insumos deve ser avaliada com cautela para ser posta em prática e fundamentada em alguns critérios preconizados pela literatura, tais como: rigorosa técnica asséptica; conservação da seringa reencapada em sua embalagem original, em local limpo e seco, junto ao frasco de insulina na geladeira ou em temperatura ambiente; aspiração de pequena quantidade de ar para prevenir a obstrução da agulha; não lavar a seringa e a agulha e nem higienizá-las com álcool [4,15].

Além disso, a pessoa com DM deverá ser avaliada pelo profissional de saúde para assegurar a ausência de infecção nos locais de aplicação de insulina, acuidade visual preservada, destreza manual e ausência de tremor, de modo que a pessoa seja capaz de recolocar o protetor da agulha com segurança $[4,15]$. Essa prática é contraindicada para indivíduos com higiene pessoal precária, infecção aguda concorrente, lesão aberta nas mãos, ou diminuição de resistência imunológica [15].

Ressalta-se que, embora controversa, a prática da reutilização é uma realidade cotidiana das pessoas com DM $[22,24,26]$ e aponta para a necessidade de se desenvolverem estudos prospectivos de avaliação dos riscos, visto que a maioria das pessoas desconhece os riscos da reutilização. De qualquer forma, a educação em DM, centrada nas necessidades individuais e nos recursos de cada pessoa, favorecerá a tomada de decisão [4].

Em relação ao descarte de seringas e agulhas, destaca-se que 18 (42,9\%) participantes o realizavam de forma inadequada, ou seja, diretamente no lixo doméstico, resultado este semelhante ao de outros estudos [22,24,26,27]. Estudo brasileiro publicado em 1999, cujo objetivo foi identificar como os usuários de insulina descartavam seringas e agulhas em seus domicílios, já mostrava descarte inadequado desses insumos [28]. Isso sugere que, ao longo das duas últimas décadas, essa prática permaneceu e, por essa razão, ainda se constitui em motivo de preocupação. $O$ descarte inadequado pode trazer danos ao meio ambiente e à saúde das pessoas [24], especialmente aquelas que trabalham com a coleta de lixo [27].

Para minimizar os riscos à saúde e ao meio ambiente, é recomendado que todo o material perfurocortante e/ou com fluidos orgânicos seja descartado em recipientes de plástico rígido, com boca larga e tampa rosqueável $[4,16,22,26]$ devendo eles serem encaminhados aos serviços de saúde para posterior incineração [4]. Destaca-se, também, a necessidade de orientação pelos profissionais de saúde às pessoas com DM para o manejo adequado dos resíduos, uma vez que a falta de informação pode fazer com que as pessoas ignorem ou subestimem a importância do descarte adequado $[22,26,27]$.

Outra importante habilidade de autocuidado relacionada à terapêutica medicamentosa é o reconhecimento dos sinais e sintomas da hipoglicemia, bem como sua prevenção e tratamento, visto que ela se constitui em uma complicação aguda decorrente do uso de insulina exógena e de AAOs secretagogos de insulina [4,14].

Dos 46 participantes, dois $(4,3 \%)$ relataram que não sabem reconhecer os sinais e sintomas da hipoglicemia, e três $(6,5 \%)$ afirmaram que nunca a sentiram, resultados estes semelhantes aos de um estudo de revisão sobre hipoglicemia, no qual somente $5 \%$ dos indivíduos com DM2 apresentaram conhecimento apropriado sobre esta condição [14]. Embora a sua ocorrência desencadeie uma série de mecanismos contrarreguladores, tais como diminuição da secreção de insulina e liberação de glucagon, adrenalina, cortisol e hormônio do crescimento, responsáveis pelo aparecimento dos sintomas, algumas pessoas podem apresentar hipoglicemias assintomáticas. Isso se deve a defeitos na resposta contrarregulatória, especialmente por insuficiência autonômica, decorrente do processo de envelhecimento e do longo tempo de doença $[4,30]$, como é o caso da amostra estudada. 
É fundamental que a pessoa com DM seja orientada quanto à possibilidade de ocorrência da hipoglicemia, a seus sinais e sintomas, bem como em relação à automonitorização da glicemia para avaliar as flutuações glicêmicas, uma vez que a ausência dos sintomas de alarme pode "inibir" o ato de comer e predispor a pessoa a maior risco de hipoglicemia grave [4,29-31].

Quanto à prevenção e ao tratamento da hipoglicemia, 17 (37\%) e cinco $(10,9 \%)$ participantes declararam, respectivamente, que não sabem preveni-la e nem tratá-la. A prevenção pode ser feita com fracionamento da alimentação, ou seja, ingerir de quatro a seis refeições diárias, sendo três refeições principais (café da manhã, almoço e jantar) e duas ou três refeições "pequenas" (lanche da manhã, da tarde e da noite). Para facilitar o fracionamento, é importante estabelecer horários para cada refeição, mesmo para os lanches, evitando ao máximo, "pular" refeições. Isso auxiliará a pessoa com DM a manter maior estabilidade glicêmica $[4,30]$.

O tratamento consiste na ingestão de 15 gramas de carboidrato simples, preferencialmente, glicose. Caso não seja possível a ingestão de tabletes de glicose, poderá ser oferecida uma colher de sopa de açúcar ou mel, $150 \mathrm{ml}$ de suco de laranja ou $150 \mathrm{ml}$ de refrigerante comum. Após 15 minutos, deve-se reavaliar a glicemia por meio de glicosímetro e, na ausência de melhora, repetir o tratamento. Cabe ressaltar que não se devem oferecer alimentos à pessoa inconsciente devido ao risco de broncoaspiração. Nessa situação, o tratamento extra-hospitalar de escolha é o glucagon, na dose de $1 \mathrm{mg}$ por via subcutânea que poderá ser repetida em 10 minutos, se não houver melhora [4].

A educação em DM é a principal estratégia para fornecer as orientações necessárias à prevenção e reversão da hipoglicemia. As ações educativas e assistenciais incluem: tratamento imediato diante da suspeita de hipoglicemia; orientação da própria pessoa, seus familiares e pessoas mais próximas, pois, na vigência de episódios moderados a graves, poderá ocorrer prejuízo da função cognitiva, o que demanda a assistência de outra pessoa para a detecção e tratamento; manutenção do cartão de identificação, uma vez que os sintomas da hipoglicemia podem se assemelhar aos de embriaguez; monitorização, quando há glicosímetro disponível, de três a quatro vezes por dia e na presença dos sintomas de hipoglicemia; alimentação nos horários determinados, com lanche noturno; programação da atividade física; atenção à prescrição medicamentosa para prevenir erros de dosagem; evitar a ingestão de bebida alcóolica; e manter consigo fontes de carboidrato simples, de fácil absorção $[4,30]$.

Não foram identificados estudos que tenham avaliado aspectos relativos à hipoglicemia entre amostras brasileiras de pessoas com DM, o que mostra a necessidade premente de se incluir esta temática nos programas educativos sobre a doença.

Como limitações da presente investigação, destacam-se o reduzido tamanho amostral, o delineamento transversal que não permite generalizações, a escassez e a heterogeneidade de estudos em amostras populacionais brasileiras que avaliaram distintos aspectos da insulinoterapia, limitando a discussão desses achados.

O desenvolvimento de habilidades para o autocuidado do DM, sobretudo as relacionadas à terapêutica medicamentosa, é essencial para manter um adequado controle metabólico e, consequentemente, prevenir e/ou retardar o aparecimento de complicações. Os dados do presente estudo mostram fragilidades no desenvolvimento de algumas dessas habilidades, destacando-se o desconhecimento sobre o horário de tomada dos AAOs, especialmente o das sulfonilureias; a dependência de outra pessoa para os procedimentos de preparo e/ou administração da insulina devido ao medo e/ou à insegurança; os erros na aspiração da dosagem correta de insulina; os locais inadequados para a aplicação da insulina e a ausência e rodízio; o armazenamento inadequado de insulina no domicílio; e a reutilização e o descarte inadequados de seringas e agulhas.

O tratamento medicamentoso do DM é complexo e desafiador. Por essa razão, as pessoas com DM devem ser orientadas e avaliadas em seu processo de autocuidado, não somente no momento do diagnóstico, mas também durante todo o curso da doença. A consulta de enfermagem pode ser uma valiosa oportunidade para se detectar tanto as dificuldades quanto as potencialidades, de modo a habilitar as pessoas e seus familiares para o cuidado do DM.

Nessa perspectiva, reitera-se a necessidade de os profissionais de saúde, especialmente os enfermeiros, implementarem atividades educativas sistematizadas, considerando as necessidades individuais e os recursos disponíveis nos serviços de saúde. 
À Pró-Reitoria de Cultura e Extensão Universitária da Universidade de São Paulo (USP), por meio do Programa Aprender com Cultura e Extensão.

1. American Diabetes Association. Introduction: Standards of medical care in diabetes mellitus-2019. Diabetes Care 2019;42(Supplement 1):S1-S2.

https://doi.org/10.2337/dc19-sint01

2. Tol A, Alhani F, Shojaeazadeh D, Sharifirad G, Moazam N. An empowering approach to promote the quality of life and self-management among type 2 diabetic patients. Edu Health Promot 2015;4(13):1-8. https://doi.org/10.4103/2277-9531.154022

3. Aquino JA, Baldoni NR, Flôr CR, Sanches C, Di Lorenzo Oliveira C, Alves GCS et al. Effectiveness of individual strategies for the empowerment of patients with diabetes mellitus: A systematic review with meta-analysis. Primary Care Diabetes 2018;12(2):97110. https://doi.org/10.1016/i.pcd.2017.10.004

4. Sociedade Brasileira de Diabetes. Diretrizes da Sociedade Brasileira de Diabetes 20172018. São Paulo: Clannad; 2017.

5. Carlos GB, Francisco LN, Moraes TC, Cerdeira CD, Santos GB. Análise das possíveis interações fármaco-alimento/nutriente em uma instituição asilar no sul de Minas Gerais. Rev Bras Pesq Saúde 2016;18(3):83-90. https://doi.org/10.21722/rbps.v18i3.15747

6. Faria HTG, Zanetti ML, Santos MA, Teixeira CRS. Conhecimento sobre terapêutica medicamentosa em diabetes: um desafio na atenção à saúde. Acta Paul Enferm 2009;22(5):612-7. https://doi.org/10.1590/s0103-21002009000500003

7. Zandoná T, Oliveira TB. Perfil dos pacientes diabéticos tipo 2 que utilizam antidiabéticos orais. Braz J Pharm 2012;93(4):476-80.

8. Moreira RC, Cruz CFR, Valsecchi EASS, Marcon SS. Vivências em família das necessidades de cuidados referentes à insulinoterapia e prevenção do pé diabético. Rev Gaúcha Enferm 2008;29(2):283-91.

9. Stacciarini TSG, Pace AE, Haas VJ. Técnica de autoaplicação de insulina com seringas descartáveis entre os usuários com diabetes mellitus, acompanhados pela Estratégia Saúde da Família. Rev Latinoam Enferm 2009;17(4):474-80. https://doi.org/10.1590/s0104-11692009000400007

10. Stacciarini TSG, Haas VJ, Pace AE. Fatores associados à auto-aplicação da insulina nos usuários com diabetes mellitus acompanhados pela Estratégia Saúde da Família. Cad Saúde Pública 2008;24(6):1314-22. https://doi.org/10.1590/s0102311x2008000600012

11. Stacciarini TSG, Caetano TSG, Pace AE. Dose de insulina prescrita versus dose de insulina aspirada. Acta Paul Enferm 2011;24(6):789-93. https://doi.org/10.1590/s010321002011000600010

12. Meece J. Dispelling myths and removing barriers about insulin in type 2 diabetes. The Diabetes Educ 2006;32(1):9S-18S. https://doi.org/10.1177/0145721705285638

13. Ahmed US, Junaidi B, Ali AW, Akhter O, Salahuddin M, Akhter J. Treatment barriers in initiating insulin therapy in a South Asian Muslim community. Diabetic Med 2010;27:169-74. https://doi.org/10.1111/i.1464-5491.2009.02904.x

14. Amiel SA, Dixon T, Mann R, Jameson K. Hypoglycaemia in type 2 diabetes. Diabetic Med 2008;25:245-54. https://doi.org/10.1111/j.1464-5491.2007.02341.x

15. Teixeira CRS, Zanetti ML, Ribeiro KP. Reutilização de seringas descartáveis: freqüência e custos para administração de insulina no domicílio. Rev Latinoam Enferm 2001;9(5):47-54. https://doi.org/10.1590/s0104-11692001000500008

16. Frid A, Hirsch L, Gaspar R, Hicks D, Kreugel G, Liersch J, et al. New injection recommendations for patients with diabetes. Diabetes Metab 2010;36:S3-S18. https://doi.org/10.1016/s1262-3636(10)70002-1

17. Gomes LC, Coelho ACM, Gomides DS, Foss-Freitas MC, Foss MC, Pace AE. Contribution of family social support to the metabolic control of people with diabetes mellitus: A randomized controlled clinical trial. Appl Nurs Res 2017;36:68-76. https://doi.org/10.1016/i.apnr.2017.05.009

18. Figueira ALG, Gomes-Villas Boas LC, Coelho ACM, Foss-Freitas MC, Pace AE. Intervenções educativas para o conhecimento da doença, adesão ao tratamento e 
controle do diabetes mellitus. Rev Latinoam Enferm 2017;25:e2863.

https://doi.org/10.1590/1518-8345.1648.2863

19. Ringborg A, Lindgren P, Yin DD, Martinell M, Stålhammar J. Time to insulin treatment and factors associated with insulin prescription in Swedish patients with type 2 diabetes. Diabetes Metab 2010;36:198-203. https://doi.org/10.1016/j.diabet.2009.11.006

20. Tavares NUL, Bertoldi AD, Mengue SS, Arrais PSD, Luiza VL, Oliveira MA, et al. Fatores associados à baixa adesão ao tratamento farmacológico de doenças crônicas no Brasil. Rev Saude Publica 2016;50(supl2):10s. https://doi.org/10.1590/s15188787.2016050006150

21. Gallo A, Pimazoni-Netto A, Zach P, Couto S, Zanella MT. Quantitative evaluation of compounded errors during insulin administration: A multicentric Brazilian study. Curr Res Diabetes Obes J 2018; 9(1):555752. https://doi.org/10.19080/crdoj.2018.09.555752

22. Vianna MS, Silva PAB, Nascimento CV, Soares SM. Self-care competence in the administration of insulin in older people aged 70 or over. Rev Latinoam Enferm 2017;25:e2943. https://doi.org/10.1590/1518-8345.2080.2943

23. Castro ARV, Grossi, SAA. Reutilização de seringas descartáveis no domicílio de crianças e adolescentes com diabetes mellitus. Rev Esc Enferm USP 2007;41(2):18795. https://doi.org/10.1590/s0080-62342007000200003

24. Stacciarini TSG, Pace AE, Iwamoto HH. Distribuição e utilização de seringas para aplicação de insulina na Estratégia Saúde da Família. Rev Eletr Enf 2010;12(1):47-55. https://doi.org/10.5216/ree.v12i1.6387

25. Agência Nacional de Vigilância Sanitária (BR). Resolução no 2.606, de 11 de agosto de 2006. Estabelece a lista de produtos médicos enquadrados como de uso único proibidos de ser reprocessados [online]. [citado 2012 Fev 27]. Disponível em: http://elegis.anvisa.gov.br/leisref/public/showAct.php?id=23407\&word

26. Cunha GH, Barbosa RVA, Fontenele MSM, Lima MAC, Franco KB, Fechine FV. Resíduos de insulinoterapia produzidos no domicílio de diabéticos acompanhados na Atenção Primária. Rev Bras Enferm 2017;70(3):618-25. https://doi.org/10.1590/00347167-2016-0406

27. Tapia CEV. Diabetes mellitus e o descarte de seringas e agulhas. Rev Gaúcha Enferm 2009;30(2):228-34.

28. Zanin STM, Carvalho WO. Diabetes mellitus e o uso domiciliar de seringas de insulina: uma questão social. Arq Ciênc Saúde Unipar 1999;3(3):205-9.

29. Teixeira CRS, Zanetti ML, Landim CAP, Becker TAC, Santos ECB, Franco RC et al. Automonitorização da glicemia capilar no domicílio: revisão integrativa da literatura. Rev Eletr Enf 2009;11(4):1006-17. https://doi.org/10.5216/ree.v1114.33257

30. Seaquist ER, Anderson J, Childs B, Cryer P, Dagogo-Jack S, Fish L et al. Hypoglycemia and diabetes: a report of a workgroup of the American Diabetes Association and the endocrine society. J Clin Endocrinol Metab 2013;98(5):1845-59. https://doi.org/10.1210/jc.2012-4127

31. Veras VS, Teixeira CRS, Santos MA, Torquato MTCG, Rodrigues FFL, Zanetti ML. Perfil glicêmico de pessoas com Diabetes mellitus em um programa de automonitorização da glicemia capilar no domicílio. Texto Contexto Enferm 2014;23(3):609-16. https://doi.org/10.1590/0104-07072014002610012 